\title{
Free Energies for Acid Attack Reactions of Lithium Cobaltate
}

\author{
R. Benedek ${ }^{\mathrm{a}, *, \mathrm{z}}$ and A. van de Walle ${ }^{\mathrm{b}}$
}

${ }^{a}$ Argonne National Laboratory, Chemical Sciences and Engineering Division, Argonne, Illinois 60439, USA

${ }^{b}$ Engineering and Applied Science Division, California Institute of Technology, Pasadena, California 91125, USA

\begin{abstract}
The attack of lithium-ion battery cathodes by stray aqueous HF, with resultant dissolution, protonation, and possibly other unintended reactions, can be a significant source of capacity fade. We explore the calculation of reaction free energies of lithium cobaltate in acid by a "hybrid" method, in which solid-phase free energies are calculated from first principles at the generalized gradient approximation + intrasite coulomb interaction $(\mathrm{GGA}+\mathrm{U})$ level and tabulated values of ionization potentials and hydration energies are employed for the aqueous species. Analysis of the dissolution of the binary oxides $\mathrm{Li}_{2} \mathrm{O}$ and $\mathrm{CoO}$ suggests that the atomic energies for $\mathrm{Co}$ and $\mathrm{Li}$ should be shifted from values calculated by first principles to yield accurate reaction free energies within the hybrid method. With the shifted atomic energies, the hybrid method was applied to analyze proton-promoted dissolution and protonation reactions of $\mathrm{LiCoO}_{2}$ in aqueous acid. Reaction free energies for the dissolution reaction, the reaction to form $\mathrm{Co}_{3} \mathrm{O}_{4}$ spinel, and the proton-for-lithium exchange reaction are obtained and compared to empirical values. An extension of the present treatment to consider partial reactions is proposed, with a view to investigating interfacial and environmental effects on the dissolution reaction.
\end{abstract}

(C) 2008 The Electrochemical Society. [DOI: 10.1149/1.2954958] All rights reserved.

Manuscript submitted September 28, 2007; revised manuscript received May 12, 2008. Published July 30, 2008.

Stray water in lithium-ion batteries reacts with organic electrolytes to generate $\mathrm{HF}^{1,2}$ which attacks the cathode material and causes irreversible capacity losses. ${ }^{3-5}$ Most of the families of cathode materials of greatest current interest, including spinel and layered systems, dissolve in acid, with $\mathrm{Li}-\mathrm{Mn}$ spinel, $\mathrm{LiMn}_{2} \mathrm{O}_{4}$, showing the highest dissolution rate. ${ }^{3}$ Acid-promoted reactions can also be beneficial, e.g., in the processing of composite cathode materials $^{6-8}$ and to leach spent cathode materials for metal recycling. ${ }^{9}$

Remedies have been implemented to mitigate the effects of acid attack in lithium-ion batteries. ${ }^{3,10-12}$ Little theoretical analysis has been performed, however, to gain a more fundamental understanding of the reactions or as a tool to screen materials for their acidattack-resistant qualities. Most desirable, in principle, would be the calculation of absolute reaction rates, which are controlled to a large extent by kinetic factors. Unfortunately, despite a long history of kinetic models, ${ }^{13}$ the prediction of oxide dissolution rates from first principles ${ }^{14}$ is still a distant prospect. The premise of this work is that the reaction free energy $\Delta G_{\mathrm{r}}$, which is more accessible to computation, ${ }^{15}$ may still provide useful guidance, even though it does not enable the prediction of dissolution rates.

Accurate calculations of $\Delta G_{\mathrm{r}}$ are readily done for reactions for which empirically derived free energies for each reaction species are available. The National Institute of Standards and Technology-Joint Army-Navy-Air Force compilation of formation free energies for ions in aqueous solution and for solid compounds, ${ }^{16}$ for example, is convenient for this purpose. Formation free energies for several of the multicomponent electrode materials of interest for lithium-ion batteries, particularly at nonideal stoichiometries, however, are not available in the standard tabulations, and for such systems an alternative approach is necessary.

We propose here to calculate reaction free energies based on a "hybrid" approach in which first-principles treatment is employed for solid phases and empirical quantities are tabulated for aqueous species. In the present work, first-principles density functional theory calculations at the GGA + U level as implemented in the Vienna ab-initio simulation package (VASP) code, ${ }^{17-19}$ are applied to calculate free energies for the solid phases. First-principles calculations have the advantage of being applicable to any material for which the atomic structure is known, and their accuracy for both molecules and solids has been improving over many years. ${ }^{20,21}$

* Electrochemical Society Active Member.

${ }^{\mathrm{z}}$ E-mail: benedek@anl.gov
Moreover, solid-phase free-energy calculations are becoming more widespread. $^{22}$ We note, however, that hybrid calculation of $\Delta G_{\mathrm{r}}$ makes especially high demands on the absolute accuracy of firstprinciples total-energy calculations because of the lack of error cancellation.

Calculations of the relatively simple dissolution reactions of the binary oxides $\mathrm{Li}_{2} \mathrm{O}$ and $\mathrm{CoO}$ enable us to calibrate the accuracy of the hybrid method. We find that the atomic reference energies for $\mathrm{Li}$ and $\mathrm{Co}$, which arise in the formulation of the hybrid method, must be shifted from their nominal values (obtained from first-principles calculations) to obtain reaction free energies consistent with those calculated from empirical-formation free energies.

The prominent lithium-ion-battery cathode material $\mathrm{Li}_{x} \mathrm{CoO}_{2}$ in acid was selected as a model system for application of the hybrid method and to test the transferability of the shifted atomic reference energies. The availability in the literature of empirical-formation free energies of $\mathrm{LiCoO}_{2}{ }^{23}$ and $\mathrm{CoO}_{2}{ }^{24}$ facilitates such tests.

Reaction free energies $\Delta G_{\mathrm{r}}$ for several hypothetical reactions of $\mathrm{Li}_{x} \mathrm{CoO}_{2}$ are calculated, including dissolution, spinel formation, and protonation. Explicit calculations are done with $x=1, \mathrm{pH} 0$, and ambient temperature and pressure; however, treatment of $\mathrm{pH}$ and $x$ as variables is straightforward, as in the Pourbaix diagram construction. ${ }^{25}$ Results at $\mathrm{pH} 0,1 \mathrm{bar}$, and room temperature are "standard" values, ${ }^{16}$ denoted by a superscript zero $\left(\Delta G_{\mathrm{r}}^{0}\right)$.

Before addressing the formal aspects of the hybrid method and their numerical application, we review in the following section some standard reactions for lithium cobaltate in acid.

\section{Reactions of Lithium Cobaltate in Acid}

A proton-promoted aqueous reaction with oxide $\mathrm{Li}_{x} \mathrm{MO}_{2}$ that results in a single oxide product phase can be written as

$$
a \mathrm{H}^{+}+b \mathrm{Li}_{x} \mathrm{MO}_{2} \rightarrow c \mathrm{H}_{\mathrm{s}} \mathrm{Li}_{\mathrm{r}} \mathrm{M}_{\mathrm{q}} \mathrm{O}_{\mathrm{p}}+d \mathrm{M}^{z+}+f \mathrm{Li}^{+}+h \mathrm{H}_{2} \mathrm{O}
$$

where the coefficients $(a, \ldots, h ; p, \ldots, x)$ are constrained by mass and charge balance; additional possibilities arise if more than one oxide product is allowed. In the following sections we review some possible reactions of $\mathrm{Li}_{x} \mathrm{CoO}_{2}$ in acid that have been proposed in the literature.

Dissolution.-Dissolution of $\mathrm{Li}_{x} \mathrm{CoO}_{2}$, accompanied by disproportionation, ${ }^{26}$ with half of the $x \mathrm{Co}^{3+}$ ions oxidized to the tetravalent state in the cobalt dioxide product and the other half solvated as $\mathrm{Co}^{2+}$, can be written, in idealized form, as 


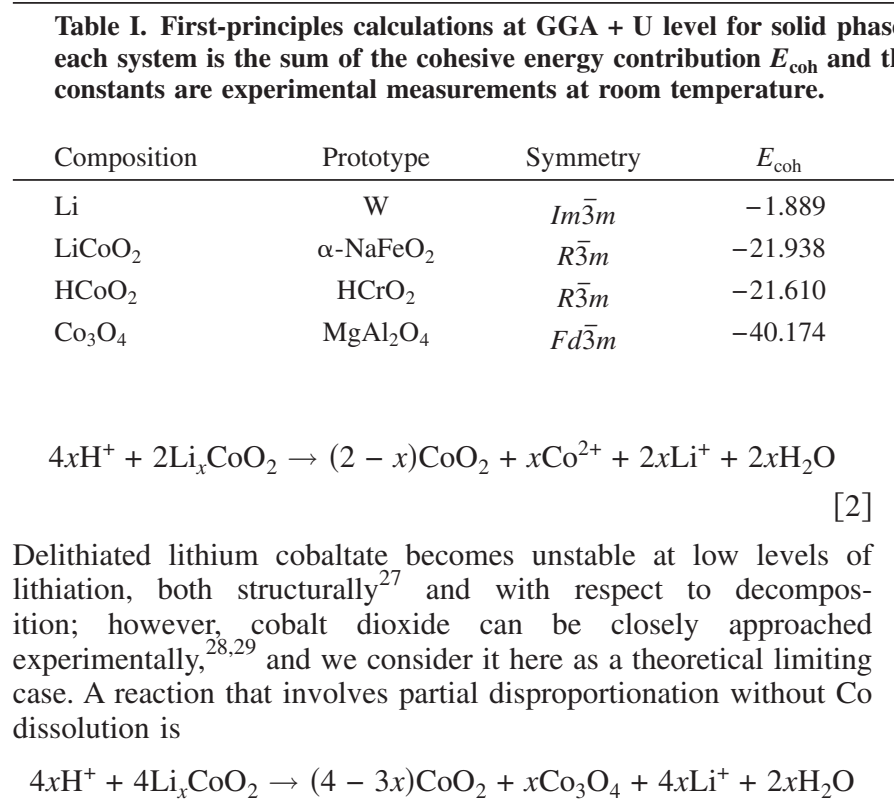

The generation of $\mathrm{Co}_{3} \mathrm{O}_{4}$ in a water-contaminated cell has been reported. ${ }^{30}$ Reaction 3 can be regarded as a step toward full disproportionation, which would occur upon subsequent dissolution of $\mathrm{Co}_{3} \mathrm{O}_{4}$

$$
4 \mathrm{H}^{+}+\mathrm{Co}_{3} \mathrm{O}_{4} \rightarrow \mathrm{CoO}_{2}+2 \mathrm{Co}^{2+}+2 \mathrm{H}_{2} \mathrm{O}
$$

Thus, the sequence of Reactions 3 and 4 is equivalent to Reaction 2. A more direct dissolution reaction than Eq. 2

$$
4 x \mathrm{H}^{+}+\mathrm{Li}_{x} \mathrm{CoO}_{2} \rightarrow(1-x) \mathrm{CoO}_{2}+x \mathrm{Co}^{3+}+x \mathrm{Li}^{+}+2 x \mathrm{H}_{2} \mathrm{O}
$$

would require the solvation of trivalent $\mathrm{Co}$ and is not considered here.

Protonation.- Another proton-promoted reaction is the ion exchange of protons with lithium. Cobalt oxyhydroxide, ${ }^{31} \mathrm{HCoO}_{2}$, is generated by the ion-exchange reaction

$$
\mathrm{H}^{+}+\mathrm{LiCoO}_{2} \rightarrow \mathrm{HCoO}_{2}+\mathrm{Li}^{+}
$$

Ligand-ion attack.- Reactions other than the proton-promoted reactions (Eq. 2-6) are possible, for example, with the fluorine derived from $\mathrm{HF}$ and the electrolyte $\mathrm{LiPF}_{6} .{ }^{32}$ Dissolution reactions are known to be promoted by contact with polyvinylidene fluoride binder in composite electrodes,${ }^{30}$ highlighting the reactions of fluorine. The present analysis, however, is restricted to proton-promoted reactions.

\section{Formulation of Hybrid Method}

We outline here the proposed hybrid method for the calculation of the reaction free energy $\Delta G_{\mathrm{r}}$, which is applied to analyze the lithium cobaltate reactions $2,3,4$, and 6 . We express the reaction free energy as

$$
\Delta G_{\mathrm{r}}=\Delta G_{\mathrm{r}}(s)+\Delta G_{\mathrm{r}}(\mathrm{aq})
$$

where the first term accounts for the solid-phase contributions and the second for contributions of aqueous species, both of which are treated as bulk properties. The prefix " $\Delta$ " denotes a difference between energies of product species and reactant species. Interfacial contributions, e.g., capacitive double- or triple-layer terms at the electrode-aqueous solution interface, may be present in principle; however, they are neglected here.

Solid-phase free energy.- We express the free energy, $G\left(s_{\mathrm{i}}\right)$, for solid phase $s_{\mathrm{i}}$, as

$$
G\left(s_{\mathrm{i}}\right)=E_{\text {coh }}\left(s_{\mathrm{i}}\right)+G_{\mathrm{vib}}(T)
$$

where $E_{\text {coh }}\left(s_{\mathrm{i}}\right)$ is a modified cohesive energy of compound $s_{\mathrm{i}}$, and $G_{\text {vib }}(T)$, the vibrational free energy, contains the zero-point vibrational energy, $E_{\mathrm{zp}}$, as well as the phonon-occupation contribution to the free energy at temperature $T$. The modified cohesive energy, which is the energy listed in the output of the VASP code, can be written as $E_{\text {coh }}\left(s_{\mathrm{i}}\right) \equiv E_{\text {tot }}\left(s_{\mathrm{i}}\right)-\Sigma v(j) E_{\text {vasp }}(j)$, where $v(j)$ is the number of $j$ atoms per formula unit of compound $s_{\mathrm{i}}$ and $E_{\mathrm{vasp}}(j)$ is the VASP atomic reference energy for atoms of type $j$.

Results of first-principles VASP calculations ${ }^{17-19}$ at the GGA $+\mathrm{U}$ level for the solid phases other than $\mathrm{CoO}_{2}$ (see the Appendix), represented in Reactions 2-6, are listed in Table I. The selection of GGA + U to represent transition-metal oxides is motivated by the high accuracy of predicted Li-ion battery cell voltages. ${ }^{33,34}$ The effective on-site interaction $U-J$ (where $U$ and $J$ are the onsite coulomb and exchange interaction parameters) for Co was taken to be $5 \mathrm{eV} .{ }^{35}$ We employ the Perdew-Wang 1991 (PW91) exchangecorrelation functional $^{36}$ for both solid phase and neutral atom calculations.

Vibrational free energy.- Although the vibrational free energy is relatively small at room temperature and pressure, it is on the order of a few tenths of an electronvolt, not entirely negligible. Furthermore, hydrogen-bearing compounds, for example, in Reaction 6, typically have large vibrational energies.

Calculations of vibrational free energies, $G_{\text {vib }}(T)$, were performed for $\mathrm{Li}, \mathrm{LiCoO}_{2}, \mathrm{HCoO}_{2}$, and $\mathrm{Co}_{3} \mathrm{O}_{4}$ using codes developed by one of the authors. ${ }^{37-39}$ The lattice dynamical analysis employs spring constants fitted to first-principles calculations of the reaction forces for small atomic displacements in a supercell geometry. ${ }^{40}$ Calculated vibrational free energies are plotted in Fig. 1. Numerical

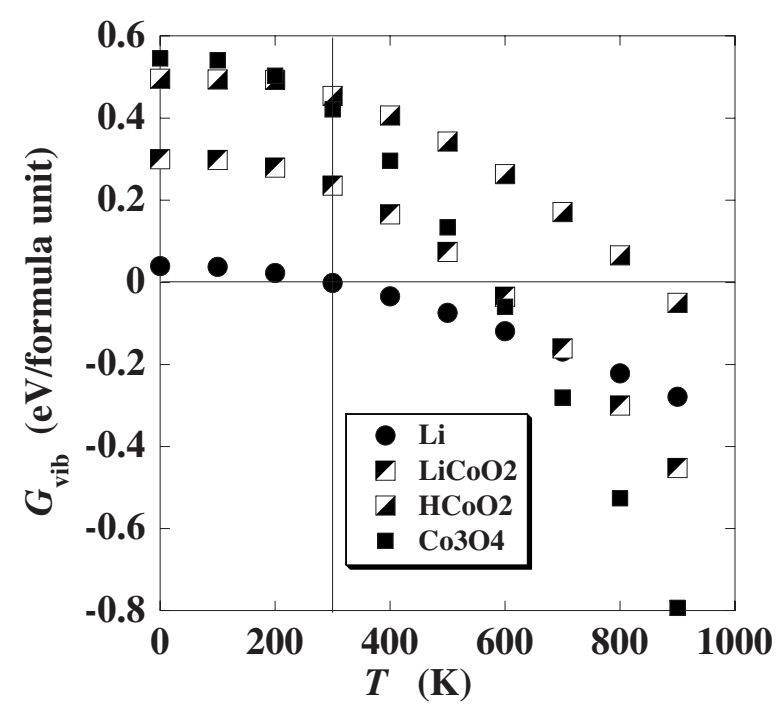

Figure 1. Calculated vibrational free energies as a function of temperature in $\mathrm{K}$. The positive free energies at low temperatures reflect the dominance of zero-point energies and low phonon occupation numbers. 
Table II. Free-energy contributions for aqueous species.

\begin{tabular}{lcccc} 
Species & $E_{\text {ref }}\left([\mathrm{aq}]_{\mathrm{i}}\right)$ & $E_{\text {ion }}\left([\mathrm{aq}]_{\mathrm{i}}\right)$ & $G_{\text {hyd }}^{0}\left([\mathrm{aq}]_{\mathrm{i}}\right)$ & $G^{0}\left([\mathrm{aq}]_{\mathrm{i}}\right)$ \\
\hline $\mathrm{H}^{+}$ & -1.116 & 13.60 & -11.45 & 1.034 \\
$\mathrm{Li}^{+}$ & -0.27 & 5.39 & -5.488 & -0.368 \\
$\mathrm{Co}^{2+}$ & -1.372 & 24.94 & -20.902 & 2.67 \\
$\mathrm{H}_{2} \mathrm{O}$ & -14.28 & & -0.266 & -14.542
\end{tabular}

values at $300 \mathrm{~K}$ are listed in Table I. The oxides $\mathrm{LiCoO}_{2}, \mathrm{HCoO}_{2}$, and $\mathrm{Co}_{3} \mathrm{O}_{4}$ were treated within the harmonic approximation, with thermal expansion neglected. The quasi-harmonic approximation was applied in the case of Li to properly account for the large thermal expansion coefficient and its appreciable effect on the free energy.

Calculations for each material were done for a series of interatomic-force-constant ranges. A range of 3-3.5 $\AA$ was found to be satisfactory for the oxides. Zero-point vibrations dominate $G_{\text {vib }}(T)$ at low temperatures. Calculated vibrational free energies for $\mathrm{LiCoO}_{2}$ at $300 \mathrm{~K}$ agree closely with estimates based on Debye and Einstein temperatures derived from calorimetry ${ }^{41}$ which yield a value of $G_{\text {vib }}=0.296 \mathrm{eV}$. This empirically derived value differs by only a few hundredths of an electronvolt from the theoretical value listed in the table, $0.253 \mathrm{eV}$.

The calculation of vibrational free energies for hydrogen-bonded systems, such as $\mathrm{HCoO}_{2}$, is complicated by a shallow double well in the potential energy surface ${ }^{31}$ associated with $\mathrm{H}$ atom motion. Calculations for $\mathrm{H}$ at the unstable equilibrium at the center of the double well yield energies about $3 \mathrm{meV}$ higher than in a site displaced by about $0.15 \AA$ toward either of the oxygens, which results in large anharmonic corrections to the potential energy. Relatively minute atomic displacements are used to determine harmonic force constants, which tends to magnify the effect of numerical noise in the calculated forces. The oscillation of protons between the doublewell minima is an additional source of configurational entropy (of the order $k \ln 2$ ), not included in the calculations.

Aqueous species free energy.-We express the free energy, $G\left(\mathrm{aq}_{\mathrm{i}}\right)$, for aqueous species $\mathrm{aq}_{\mathrm{i}}$ as

$$
G\left(\mathrm{aq}_{\mathrm{i}}\right)=E_{\text {ref }}\left(\mathrm{aq}_{\mathrm{i}}\right)+E_{\text {ion }}\left(\mathrm{aq}_{\mathrm{i}}\right)+G_{\text {hyd }}\left(\mathrm{aq}_{\mathrm{i}}, T\right)
$$

where $E_{\mathrm{ref}}\left(\mathrm{aq}_{\mathrm{i}}\right)$ is the energy of an isolated neutral atom of type $i$, $E_{\text {ion }}\left(\mathrm{aq}_{\mathrm{i}}\right)$ is the sum of ionization potentials of $\left(\mathrm{aq}_{\mathrm{i}}\right)$ for oxidation state $z\left(\mathrm{aq}_{\mathrm{i}}\right)$, and $G_{\text {hyd }}\left(\mathrm{aq}_{\mathrm{i}}, T\right)$ is the hydration free energy. Ionization potentials were taken from www.camdb.ac.cn (this website provides an Atomic \& Molecular Database compiled by the Institute of Applied Physics and Computational Mathematics (IAPCM) and the Chinese National Committee for CODATA) and hydration free energies from Fawcett. ${ }^{42}$ Numerical values are listed in Table II.

Because the VASP atomic reference energy, $E_{\mathrm{vasp}}(j)$, differs from the atomic ground state energy, a correction must be made to reconcile $\Delta G(s)$ and $\Delta G(\mathrm{aq})$. A VASP calculation for neutral atom $i$ in its ground state yields the energy $E_{\mathrm{gr}}(i)-E_{\text {vasp }}(i) \equiv E_{\text {ref }}\left(\mathrm{aq}_{\mathrm{i}}\right)$. As described below, $E_{\mathrm{gr}}$ is shifted to achieve consistency between reaction free energies calculated with the hybrid method and from empiricalformation free energies. The atomic reference energies, $E_{\mathrm{ref}}\left(\mathrm{aq}_{\mathrm{i}}\right)$, listed in Table II, are calculated at the GGA level.

\section{Hybrid-Method Reaction Free Energy Calculations}

Accuracy.- The accuracy of hybrid-method calculations of $\Delta G_{\mathrm{r}}$ depends on the ability of first-principles calculations to represent energy differences for vastly different states of matter: the reactant solid phase energy, $G\left(s_{\mathrm{i}}\right)$, and the product aqueous phase energy, $G\left(\mathrm{aq}_{\mathrm{i}}\right)$, which are subtracted from each other to obtain $\Delta G_{\mathrm{r}}$ in Eq. 7. In effect, any error in the chemical potential difference, $\mu_{\mathrm{i}}(s)$ $-\mu_{\mathrm{i}}(a t)$, between species $i$ in a solid phase and in the neutral atom is reflected directly in the error in the calculated $\Delta G_{\mathrm{r}}$. The present

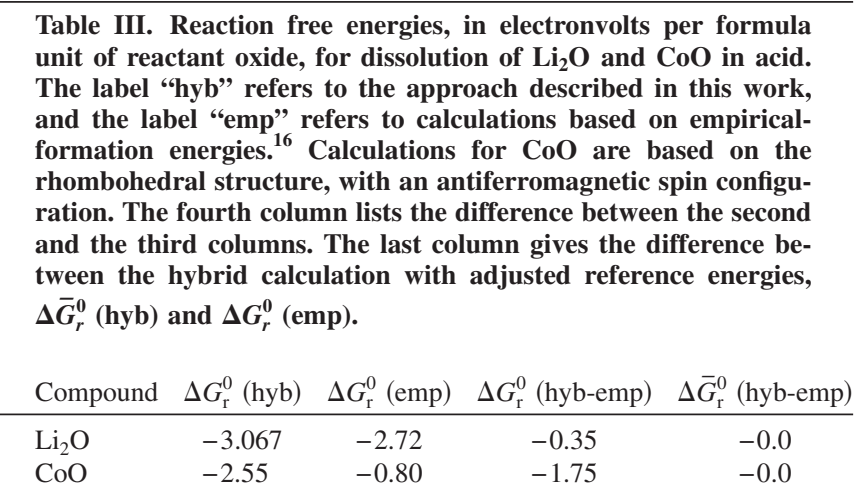

application, in common with other types of chemical reactions that involve disparate states of matter, ${ }^{43}$ therefore cannot rely on the cancellation of errors that occurs when energy differences are taken between relatively similar states of matter. In the present work, we adopt the remedy described in the next section to enhance the accuracy of the calculated $\Delta G_{\mathrm{r}}$.

Dissolution of $\mathrm{Li}_{2} \mathrm{O}$ and $\mathrm{CoO}$.- Application of the hybrid method to binary oxide dissolution enables us to calibrate the method. We consider the dissolution reactions

$$
2 \mathrm{H}^{+}+\mathrm{Li}_{2} \mathrm{O} \rightarrow 2 \mathrm{Li}^{+}+\mathrm{H}_{2} \mathrm{O}
$$

and

$$
2 \mathrm{H}^{+}+\mathrm{CoO} \rightarrow \mathrm{Co}^{2+}+\mathrm{H}_{2} \mathrm{O}
$$

which are analogous to Reactions 2-5 for $\mathrm{Li}_{x} \mathrm{CoO}_{2}$. Predictions of $\Delta G_{\mathrm{r}}^{0}$ based on standard formation-free-energy tabulations ${ }^{16}$ and based on the hybrid method are listed in Table III.

The hybrid method predicts reaction free energies, $\Delta G_{\mathrm{r}}^{0}$, that are lower (more energetically favorable) than the empirical-formation energy predictions, considerably more so for $\mathrm{CoO}$ than for $\mathrm{Li}_{2} \mathrm{O}$. If we assume, for simplicity, that the discrepancy between the predicted binary-oxide-dissolution-reaction free energies from empirical-formation energies and by the hybrid method result entirely from errors in $E_{\text {ref }}(\mathrm{Co})$ and $E_{\text {ref }}(\mathrm{Li})$, then the two methods can be essentially reconciled by adopting corrections $\delta E_{\text {ref }}(\mathrm{Co})$ $=1.75 \mathrm{eV}$ and $\delta E_{\text {ref }}(\mathrm{Li})=0.35 \mathrm{eV}$. With these choices, the corrected atomic reference energies (denoted by the bar) are $\bar{E}_{\text {ref }}(\mathrm{Co})$ $=0.38 \mathrm{eV}$ and $\bar{E}_{\text {ref }}(\mathrm{Li})=0.08 \mathrm{eV}$. We take $\delta E_{\text {ref }}(\mathrm{H})=0.0$, because, to a good approximation, $-E_{\text {ion }}(\mathrm{H})=E_{\text {vasp }}(\mathrm{H})+E_{\text {ref }}(\mathrm{H})$.

Reaction free energies for $\mathrm{LiCoO}_{2}$ in acid.- Free energies for Reactions 2-4 and 6, based on the shifted reference energies, are listed in Table IV. Reaction 5 is omitted, because $\mathrm{Co}^{3+}$ is not included in the hydration energy tabulation used in the analysis. ${ }^{42}$ The free energy for the delithiated cobaltate, $\mathrm{CoO}_{2}$, was obtained by an extrapolation procedure (described in the Appendix) using experimentally measured cell voltages to avoid ambiguities introduced by the metal-insulator transition that occurs in delithiated $\mathrm{Li}_{x} \mathrm{CoO}_{2}$. The last two columns of Table IV show the differences between hybridmethod calculations and empirical-formation energy calculations for Reactions 2-4 and 6; the column labeled $\Delta G_{\mathrm{r}}^{0}$ (hyb-emp) is based on unshifted reference energies $E_{\text {ref }}$, and that labeled $\Delta \bar{G}_{\mathrm{r}}^{0}$ (hyb-emp) employs adjusted reference energies, $\bar{E}_{\text {ref }}$. The agreement between hybrid-method and empirical-method calculations is considerably improved by shifting the reference energies. Some discrepancy remains in the case of the protonation Reaction 6, the source of which is not investigated further here.

\section{Discussion}

Significance of reaction free energies. - The empirical-standard free energies, $\Delta G_{\mathrm{r}}^{0}(\mathrm{emp})$, listed in Table IV, for Reactions 2-4 and 6 


\begin{tabular}{|c|c|c|c|c|c|c|}
\hline Reactant & Reaction & $\Delta G^{\circ}$ (hyb) & $\Delta \bar{G}^{\circ}$ (hyb) & $\Delta G^{\circ}(\mathrm{emp})$ & $\Delta G_{\mathrm{r}}^{\circ}($ hyb-emp) & $\Delta \bar{G}_{\mathrm{r}}^{\circ}($ hyb-emp) \\
\hline $\mathrm{LiCoO}_{2}$ & 2 (dissolution) & -1.72 & -0.50 & -0.49 & -1.23 & 0.01 \\
\hline $\mathrm{LiCoO}_{2}$ & $3\left(\mathrm{Co}_{3} \mathrm{O}_{4}\right.$ form. $)$ & -0.81 & -0.46 & -0.42 & -0.39 & 0.04 \\
\hline $\mathrm{Co}_{3} \mathrm{O}_{4}$ & 4 (dissolution) & -3.63 & -0.23 & -0.27 & -3.36 & +0.04 \\
\hline $\mathrm{LiCoO}_{2}$ & 6 (protonation) & -0.88 & -0.53 & -0.25 & -0.63 & -0.28 \\
\hline
\end{tabular}

are plotted in Fig. 2 against the mean oxidation state of cobalt in the product compounds. All the reactions have negative free energies (positive thermodynamic driving forces, $-\Delta G_{\mathrm{r}}^{0}$ ) and are thus thermodynamically allowed. The maximum free-energy decrease is achieved when disproportionation of $\mathrm{Co}^{3+}$ in the reactant $\mathrm{LiCoO}_{2}$ has occurred to yield $\mathrm{CoO}_{2}$ along with solvated $\mathrm{Co}^{2+}$. This can be achieved directly, by the dissolution of Reaction 2, or by the sequence of Reactions 3 and 4 . The driving force available for Reaction 3, in which spinel $\mathrm{Co}_{3} \mathrm{O}_{4}$ and $\mathrm{CoO}_{2}$ are produced, is only slightly less than that for Reaction 2. The observation ${ }^{30}$ of $\mathrm{Co}_{3} \mathrm{O}_{4}$ in a $\mathrm{LiCoO}_{2}$ cell is therefore plausible based on these considerations. The dashed lines in the figure, which correspond to the dissolution of either the oxyhydroxide or $\mathrm{Co}_{3} \mathrm{O}_{4}$, refer to reactions that have not been reported in the literature.

Explicit calculations were presented only for full lithiation $(x$ $=1)$. The free energies for Reactions 2,3 , and $6, \Delta G_{\mathrm{r}}^{0}(x)$, decrease to zero, per formula unit of $\mathrm{Li}_{x} \mathrm{CoO}_{2}$, as lithium is depleted to $x$ $=0$, because the reactions require either trivalent Co or protonexchangeable Li. Proton exchange has primarily been observed in Li-depleted specimens.

The preceding discussion addresses only standard free energies (denoted by the superscript zero), which correspond to $\mathrm{pH} 0$. Under more basic conditions, the driving force for Reactions 3 and 6 increases (smaller $a / b$ in the generic Reactions 1), relative to Reaction

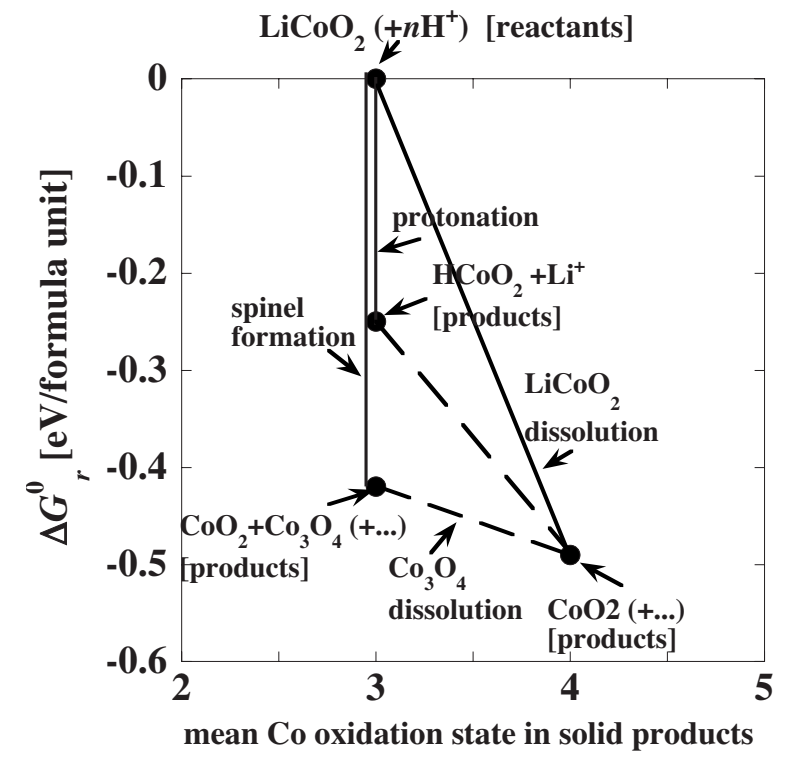

Figure 2. The relative magnitudes of reaction free energies, $\Delta G_{\mathrm{r}}^{0}$, per reacting $\mathrm{LiCoO}_{2}$ formula unit, for Reactions 2-4 and 6 are shown on the ordinate scale. [The vertical tieline for Reaction 3 (spinel formation) is shifted slightly in the figure with respect to Reaction 6 (protonation) to distinguish the two reactions.] The abscissa is the mean Co oxidation state in the product solid phase(s). The parentheses denote the aqueous species in the reaction products. Reaction 2 has the largest thermodynamic driving force, followed by Reactions 3 and 6.
2. Without knowledge of kinetic factors, of course, no inference is possible regarding reaction-rate variations with $\mathrm{pH}$ and $x$ or the relative rates of Reactions 2-4 and 6.

Effect of interface environment.- The sign of the reaction free energy provides information as to whether the reaction is thermodynamically favorable. Unfortunately, the ability to calculate this free energy, developed in this article, does not in itself enable materials to be screened for resistance to dissolution, which would be desirable for battery electrode design. Our approach has been restricted to bulk properties, but the chemistry and structure at the oxideaqueous interface would be expected to play a role in the dissolution process. Before concluding, we briefly outline how such effects might be simulated.

Several more-or-less distinct processes must take place at the surface for the dissolution Reaction 2 to proceed: solvation of $\mathrm{Co}^{2+}$, solvation of $\mathrm{Li}^{+}$, and the formation of water molecules. Although these processes cannot rigorously be treated as independent, further development of the simulation is possible only if they are regarded as at least approximately independent. Critical for the dissolution is the solvation of the transition element

$$
\mathrm{Co}\left(s_{\mathrm{r}}\right) \rightarrow \mathrm{Co}^{2+}(\mathrm{aq})+2 e^{-}
$$

Formally, we can regard the free-energy change associated with this partial reaction as

$$
\Delta G_{\mathrm{Co}^{2+}}=E_{\mathrm{ion}}\left(\mathrm{Co}^{2+}\right)+G_{\mathrm{hyd}}\left(\mathrm{Co}^{2+}\right)+\bar{E}_{\mathrm{ref}}(\mathrm{Co})+2 \mu_{\mathrm{e}}^{-}-\mu\left(\mathrm{Co}_{\mathrm{s}_{\mathrm{r}}}\right)
$$

where $\mu_{\mathrm{e}}$ is the electron chemical potential and $\mu\left(\mathrm{Co}_{\mathrm{s}_{\mathrm{r}}}\right)$ is the chemical potential of $\mathrm{Co}$ atoms in $\mathrm{LiCoO}_{2}$.

The absolute value of $\Delta G_{\mathrm{Co}^{2}+}$ is not necessarily of significance; however, relative values as a function of local chemistry and structure may suggest ways to improve resistance to acid attack. For example, models of the oxide-aqueous interface, e.g., from firstprinciples molecular-dynamics simulations, could be used to determine the detachment energy of an exposed interface atom, which can then be employed in Eq. (13) instead of the bulk chemical potential $\mu\left(\mathrm{Co}_{\mathrm{s}_{\mathrm{r}}}\right)$.

\section{Conclusion}

We propose a hybrid method, based on first-principles calculations for solid phases combined with empirical-hydration free energies, for the calculation of reaction free energies of complex oxides in aqueous acids. The method has been applied to reactions of $\mathrm{LiCoO}_{2}$ in acid. The advantage of the approach is that calculations can be performed for materials of arbitrary stoichiometry if the atomic structure is known. A technical difficulty is that effective atomic reference energies cannot be determined entirely ab initio. Nevertheless, we have demonstrated that a set of values for atomic reference energies of $\mathrm{Li}$ and $\mathrm{Co}$, obtained from an analysis of binary oxides, is able to accurately account for the energies of several reactions of $\mathrm{LiCoO}_{2}$ in acid.

Calculated reaction free energies enable assessment of the thermodynamic feasibility of a reaction. An extension of the present treatment, to decompose the net reaction into partial reactions, is 


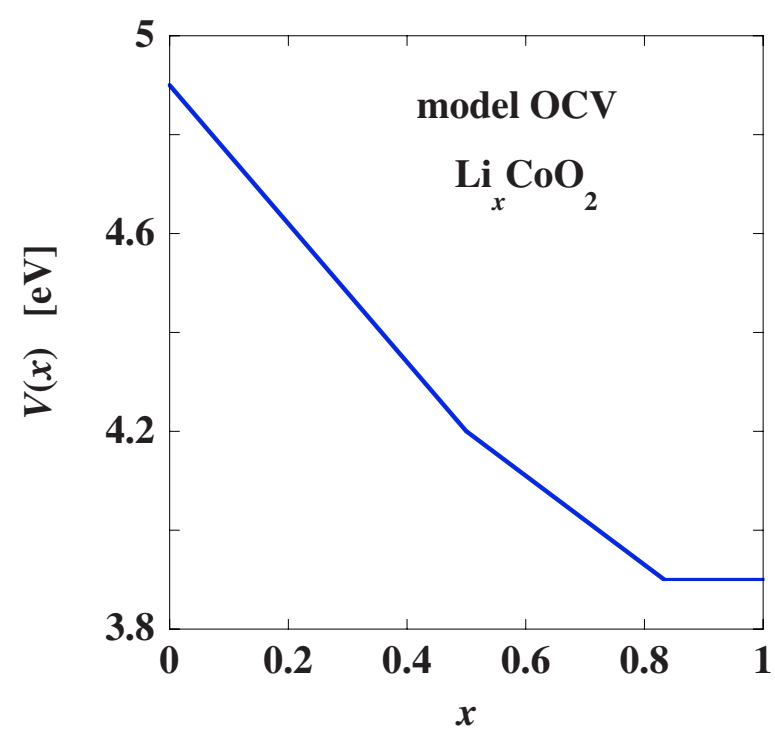

Figure A-1. (Color online) Schematic form of open-circuit cell voltage for $\mathrm{Li}_{x} \mathrm{CoO}_{2}$, in electronvolts, as a function of lithiation, employed in calculation of the free energy of $\mathrm{CoO}_{2}$, using Eq. A-1.

proposed, which may enable interfacial and environmental effects on the reaction free energy to be explored by simulation.

\section{Acknowledgments}

We are most grateful to M. M. Thackeray for his guidance and encouragement. This work was supported by the Office of FreedomCar and Vehicle Technologies (Batteries for Advanced Transportation Technologies Program), U.S. Department of Energy, under contract no. W31-109-Eng-38. A. van de Walle was supported by the National Science Foundation through TeraGrid computing resources provided by NCSA and SDSC under grant DMR060011N. Grants of computer time at the National Energy Research Supercomputer Center, Lawrence Berkeley Laboratory, are gratefully acknowledged. The submitted manuscript has been created by UChicago Argonne, LLC, Operator of Argonne National Laboratory (Argonne). Argonne, a U.S. Department of Energy Office of Science laboratory, is operated under contract no. DE-AC02-06CH11357. The U.S. Government retains for itself, and others acting on its behalf, a paid-up nonexclusive, irrevocable worldwide license in said article to reproduce, prepare derivative works, distribute copies to the public, and perform publicly and display publicly, by or on behalf of the Government.

Argonne National Laboratory assisted in meeting the publication costs of this article.

\section{Appendix Free Energy of Cobalt Dioxide}

If the free energy of $\mathrm{LiCoO}_{2}, G^{0}(x=1)$, the chemical potential of lithium metal, $\mu(\mathrm{Li})$, as well as open-circuit electrochemical cell voltages $V(x)$, with respect to a lithium metal anode, are known, the free energy $G^{0}(x)$ of $\mathrm{Li}_{x} \mathrm{CoO}_{2}$ can be obtained by the interpolation

$$
G^{0}(x)=G^{0}(1)+(x-1)[\mu(\mathrm{Li})-\bar{V}(x, 1)]
$$

where $\bar{V}(x, 1)$ is the average cell voltage in the composition interval $(x, 1)$. We can thereby determine the free energy of $\mathrm{CoO}_{2}$ without having to extend the GGA + U calculations to the metallic side of the metal-insulator transition at about $x=0.85$.
Only specification of $\mu(\mathrm{Li}), G^{0}(1)$, and $V(x)$ remains in order to implement this treatment. Calculations for $\mathrm{Li}$ in the body-centered cubic structure were performed with the projector augmented wave (PAW) implementation ${ }^{19}$ of the VASP code ${ }^{17,18}$ at the GGA level. A piecewise linear model of $V(x)$ is adopted: $V(0)=4.9 ; V(1 / 2)=4.2$; and $V(5 / 6)=V(1)=3.9$ in $\mathrm{eV}$; with linear dependences of $V(x)$ for intermediate values of $x$ (cf. Fig. A-1) ${ }^{7}$. Measured open-circuit cell voltages ${ }^{29}$ are close to the model $V(x)$.

\section{References}

1. C. G. Barlow, Electrochem. Solid-State Lett., 2, 362 (1999).

2. W. Li and B. L. Lucht, J. Electrochem. Soc., 153, A1617 (2006).

3. W. Choi and A. Manthiram, J. Electrochem. Soc., 153, A1760 (2006).

4. D. Aurbach, B. Markovsky, A. Rodkin, E. Levi, Y. S. Cohen, H. J. Kim, and M. Schmidt, Electrochim. Acta, 47, 4291 (2002).

5. Z. Chen and J. R. Dahn, Electrochem. Solid-State Lett., 7, A11 (2004).

6. Y. Paik, C. P. Grey, C. S. Johnson, J. S. Kim, and M. M. Thackeray, Chem. Mater, 14, 5109 (2002).

7. C. S. Johnson, J. S. Kim, C. Lefief, N. Li, J. T. Vaughey, and M. M. Thackeray, Electrochem. Commun., 6, 1085 (2004).

8. M. M. Thackeray, C. S. Johnson, J. T. Vaughey, N. Li, and S. A. Hackney, J. Mater. Chem., 15, 2257 (2005).

9. S. Sakultung, K. Pruksathorn, and M. Hunsom, Korean J. Chem. Eng., 24, 272 (2007).

10. Y. K. Sun, J. M. Han, S. T. Myung, S. W. Lee, and K. Amine, Electrochem Commun., 8, 821 (2006).

11. Y. K. Sun, S. W. Cho, S. W. Lee, C. S. Yoon, and K. Amine, J. Electrochem. Soc., 154, A168 (2007).

12. K. Amine, J. Liu, S. Kang, I. Belharouak, Y. Hyung, D. Vissers, and G. Henriksen, J. Power Sources, 129, 14 (2004)

13. M. A. Blesa, P. J. Morando, and A. E. Regazzoni, Chemical Dissolution of Metal Oxides, Chap. 9, CRC Press, Boca Raton, FL (1993).

14. A. C. Lasaga and A. Luttge, J. Phys. Chem. B, 109, 1635 (2005).

15. R. Benedek and M. M. Thackeray, Electrochem. Solid-State Lett., 9, A265 (2006).

16. D. D. Wagman, W. H. Evans, V. B. Parker, R. H. Schumm, I. Halow, S. M. Bailey, K. L. Churney, and R. L. Nuttall, J. Phys. Chem. Ref. Data, 11, 2 (1982).

17. G. Kresse and J. Furthmueller, Comput. Mater. Sci., 6, 15 (1996).

18. G. Kresse and J. Furthmueller, Phys. Rev. B, 54, 11169 (1996).

19. G. Kresse and D. Joubert, Phys. Rev. B, 59, 1758 (1999).

20. J. P. Perdew, J. A. Chevary, S. H. Vosko, K. A. Jackson, M. R. Pederson, D. J. Singh, and C. Fiolhais, Phys. Rev. B, 46, 6671 (1992).

21. J. P. Perdew, J. M. Tao, V. N. Staroverov, and G. E. Scuseria, J. Chem. Phys., 120, 6898 (2004).

22. E. I. Isaev, N. V. Skorodumova, R. Ahuja, Y. K. Vekilov, and B. Johansson, Proc. Natl. Acad. Sci. U.S.A., 104, 9168 (2007).

23. H. Yokokawa, N. Sakai, K. Yamaji, T. Horita, and M. Ishikawa, Solid State Ionics, 113-115, 1 (1998).

24. Y. Tao, B. Zhu, and Z. Chen, J. Alloys Compd., 430, 222 (2007).

25. ASM Handbook, S. D. Cramer and J. B. S. Covino, Editors, Chap. 2, ASM International, Materials Park, OH (2003).

26. R. Gupta and A. Manthiram, J. Solid State Chem., 121, 483 (1996).

27. A. V. der Ven, M. K. Aydinol, G. Ceder, G. Kresse, and J. Hafner, Phys. Rev. B, 58, 2975 (1998).

28. L. Daheron, R. Dedryvere, H. Martinez, M. Menetrier, C. Denage, C. Delmas, and D. Gonbeau, Chem. Mater, 20, 583 (2008).

29. G. G. Amatucci, J. M. Tarascon, and L. C. Klein, J. Electrochem. Soc., 143, 1114 (1996).

30. D. Aurbach, B. Markovsky, G. Salitra, E. Markevich, Y. Talyossef, M. Koltypin, L. Nazar, B. Ellis, and D. Kovacheva, J. Power Sources, 165, 491 (2007).

31. R. G. Delaplane, J. A. Ibers, J. R. Ferraro, and J. J. Rush, J. Chem. Phys., 50, 1920 (1969).

32. O. V. Bushkova, O. L. Andreev, N. N. Batalov, S. N. Shkerin, M. V. Kuznetsov, A. P. Tyutyunnik, O. V. Koryakova, E. H. Song, and H. J. Chung, J. Power Sources, 157, 477 (2006)

33. F. Zhou, C. A. Marianetti, C. Cococcioni, D. Morgan, and G. Ceder, Phys. Rev. B, 69, 201101 (2004).

34. F. Zhou, C. Cococcioni, C. A. Marianetti, D. Morgan, and G. Ceder, Phys. Rev. B, 70, 235121 (2004).

35. M. Cococcioni and S. de Gironcoli, Phys. Rev. B, 71, 035105 (2005).

36. Y. Wang and J. P. Perdew, Phys. Rev. B, 44, 13298 (1991).

37. A. van de Walle, M. Asta, and G. Ceder, CALPHAD: Comput. Coupling Phase Diagrams Thermochem., 26, 539 (2002).

38. A. van de Walle and G. Ceder, J. Phase Equilib., 23, 348 (2002).

39. A. van de Walle, unpublished; see www.its.caltech.edu/avdw/atat/ (2002).

40. A. van de Walle and G. Ceder, Rev. Mod. Phys., 74, 11 (2002).

41. H. Kawaji, M. Takematsu, T. Tojo, T. Atake, H. Hirano, and R. Kanno, J. Therm Anal. Calorim., 68, 833 (2002).

42. W. R. Fawcett, J. Phys. Chem. B, 103, 11181 (1999).

43. L. Wang, T. Maxisch, and G. Ceder, Phys. Rev. B, 73, 195107 (2006). 